\title{
USCT data challenge
}

Nicole Ruiter, Michael Zapf, Torsten Hopp, Hartmut Gemmeke, Koen van Dongen

Nicole V. Ruiter, Michael Zapf, Torsten Hopp, Hartmut Gemmeke, Koen W. A. van Dongen, "USCT data challenge," Proc. SPIE 10139, Medical Imaging 2017: Ultrasonic Imaging and Tomography, 101391N (13 March 2017); doi: $10.1117 / 12.2272593$

SPIE. Event: SPIE Medical Imaging, 2017, Orlando, Florida, United States 


\title{
USCT data challenge
}

\author{
Nicole V. Ruiter ${ }^{1}$, Michael Zapf ${ }^{1}$, Torsten Hopp ${ }^{1}$, Hartmut Gemmeke ${ }^{1}$, Koen W.A. van Dongen ${ }^{2}$ \\ ${ }^{1}$ Institute for Data Processing and Electronics, Karlsruhe Institute of Technology, Karlsruhe, \\ Germany \\ ${ }^{2}$ Laboratory of Acoustical Wavefield Imaging, Delft University of Technology, Delft, the \\ Netherlands
}

\begin{abstract}
In the past years we have perceived within the USCT research community a demand for freely available USCT data sets. Inspired by the idea of Open Science, this collection of data sets could stimulate the collaboration and the exchange of ideas and experiences between USCT researchers. In addition, it may lead to comprehensive comparison of different reconstruction algorithms and their results. Finally, by collecting feedback from the users about data and system architecture, valuable information is gathered for further development of measurement setups. For the above reasons, we have initiated a digital portal with several reference data sets and access scripts under free licenses. To kick off this initiative, we organized a USCT data challenge event at SPIE Medical Imaging 2017.
\end{abstract}

Keywords: Ultrasound computer tomography, data challenge, open science, open data

\section{INTRODUCTION}

Ultrasound Computer Tomography is an exciting new technology mostly aimed at breast cancer imaging. Due to the complex interaction of ultrasound with human tissue, the large amount of raw data, and the large volumes of interest, image reconstruction is challenging both from a physical and a computational point of view. Many different image reconstruction methods can be applied, ranging from simple ray based methods to full inversion with very different approximations of the linear acoustic wave equation, see e.g. [1]. While the ray based algorithms offer fast reconstruction, the inversion algorithms promise high image quality and resolution.

In recent years various groups have proposed a number of different approaches for image reconstruction, e.g. [1-23]. Direct comparison and analysis of the algorithms is difficult as they are usually applied to simulated data or - if available - to real data acquired with very different measurement setups, e.g. [24-28]. The relatively few experimental data sets are usually not commonly available. Moreover, even if they would be readily available, using the different date sets to test a new reconstruction method would require accurate (and often not freely available) knowledge about the experimental setup and data handling.

To mitigate such challenges for the scientific communities, there is an ongoing endeavor for so-called "Open science". Several leading European academic institutions signed in 2003 the "Berlin Declaration" on "open access to knowledge" to enable easier exchange, participation and collaboration within the scientific community [33-35].

Following this idea and to mend gaps within the US(C)T community, the establishment of open and easy to use data and code interfaces is the long term goal of this initiative. To stimulate the exchange of the available reconstruction algorithms and raw data sets of different USCT devices, a reference database with freely available and open licensed USCT data for comparison of reconstruction algorithms will be established, maintained and updated. Additionally, the feedback about data and system architecture of the scientists working on reconstruction methods will help to drive further development of the various measurement setups.

\section{DESCRIPTION OF CHALLENGE}

This challenge aims on applying available image reconstruction algorithms on provided USCT data in order to establish a first intercommunication and standards for open data interface. In addition, this challenge marks the start of the USCT reference database. The data sets and software for data access are available via the USCT challenge home page and the linked data and code repository [29]. The long term goals of this work is to build up a free and open licensed reference

\footnotetext{
Medical Imaging 2017: Ultrasonic Imaging and Tomography, edited by Neb Duric, Brecht Heyde, Proc. of
} SPIE Vol. 10139, 101391N · @ 2017 SPIE · CCC code: 1605-7422/17/\$18 · doi: 10.1117/12.2272593 

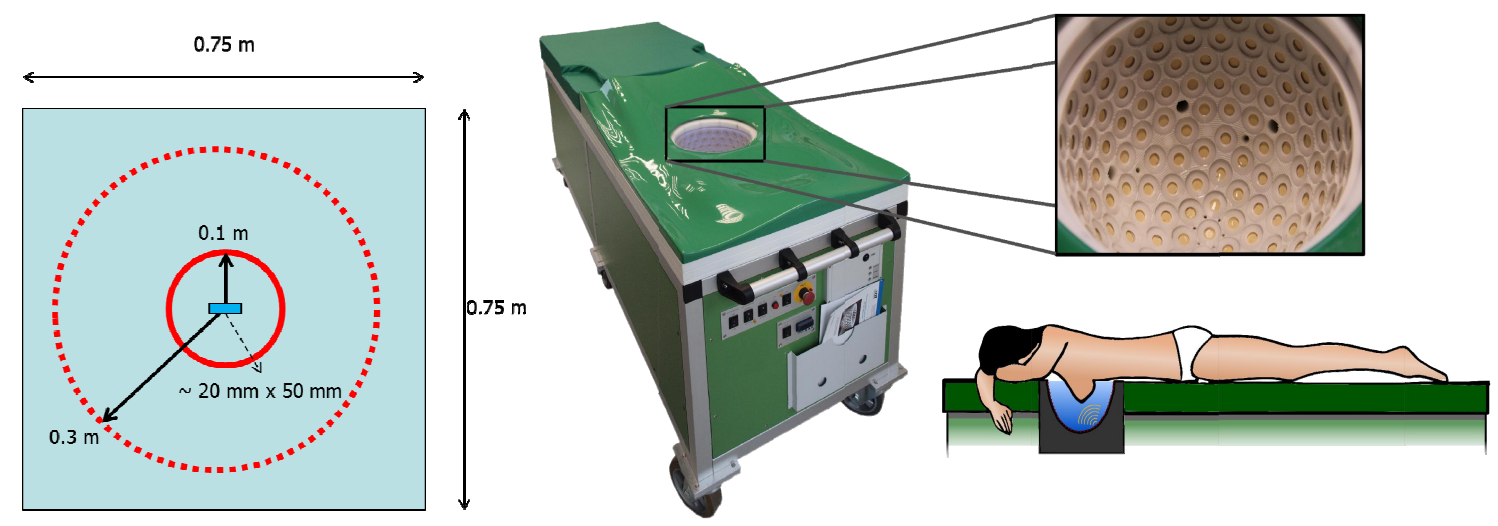

Figure 1: The two scanning systems; (left) sketch of the USCT system from the TU Delft: a water tank with a rotating object (tissue mimicking agar phantom of approx. $20 \mathrm{~mm} \times 50 \mathrm{~mm}$ ) emulating a rotating source at a radial distance of $0.3 \mathrm{~m}$ and a rotating receiver at a radial distance of $0.1 \mathrm{~m}$; (right) KIT 3D USCT: USCT with patient bed (center), transducer aperture (top right) and patient position (bottom right).

USCT data base which is available for the whole community. We expect this to enable reproducible comparison of image reconstruction algorithms and USCT systems and to establish user friendly and easy to use interfaces, standards and data formats between the different USCT systems and their reconstruction algorithms, software and data formats.

The materials are provided using a free and open license, i.e. the BSD 3-clause license [30] for code and data, allowing free use and publication of results. After the challenge the data sets may remain with the participants, and can be used for additional research and other purposes. Further publication and results should follow good academic practices and cite this paper.

The participants are encouraged to a submit field report to the SPIE Medical image conference 2017. This report is preferably based on the provided field report template and may contain information about the applied algorithm (e.g. citation of paper), and - if available - reconstructed images and performance results. The participants agree that their participation and their results may be published in connection with their names and affiliations, also under a free and open license and open access standards. The participants are also invited to share their results, feedback and suggestions at the poster session and the open panel discussion of the SPIE Ultrasonic Imaging and Tomography conference.

\section{USCT SYSTEM OF TU DELFT}

The Delft Breast Ultrasound Scanner (DBUS) is depicted on the left-hand side of Figure 1. The system consists of a water tank with dimensions $0.75 \mathrm{~m} \times 0.75 \mathrm{~m} \times 0.65 \mathrm{~m}$ with a water level of $0.45 \mathrm{~m}$. The temperature of the water is continuously monitored via thermocouples and kept constant within $1^{\circ} \mathrm{C}$ using heating mats and a temperature controller. On top of the system, two rotary stages (LG Motion LGR1090-PD), controlled by motor drivers (Parker), are mounted. The first rotary stage rotates the object, the second the receiver $(0.5 \mathrm{MHz}$, Panametrics V318). The source, which is identical to the receiver, is mounted at a fixed position in the corner of the tank. In this way a 2D tomographic scan of the object is obtained. For each A-scan, an electric pulse with a center frequency of approx. $0.5 \mathrm{MHz}$ is generated by an arbitrary wave form generator (Agilent 33250A), amplified (Electronics and Innovation 210L $40 \mathrm{~dB}$ ), and successively damped using a variable attenuator (JFW Industries, 50BR-036). The resulting wave field is measured using an identical transducer connected to a 14 bit A/D converter (Spectrum M3i.4142-exp - PCI) which is set to a sample rate of $400 \mathrm{MHz}$. For each A-scan, in addition to the raw (unfiltered) RF data, information such as temperature, source and receiver positions, etc. is stored. Together with a scan including an object, a scan in absence of an object, referred to as an empty scan, is made for reference. An example of an empty scan is shown in Figure 2. The A-scan, presented in time and frequency domain, corresponds to a measurement with the source and receiver facing each other.

\section{USCT SYSTEM OF KIT}

The KIT's 3D Ultrasound Computer Tomography system (3D USCT) is depicted on the right hand side of Figure 1. The device has a semi-ellipsoidal 3D aperture. Approx. spherical wave fronts are generated by each emitter at $2.5 \mathrm{MHz}$ and 

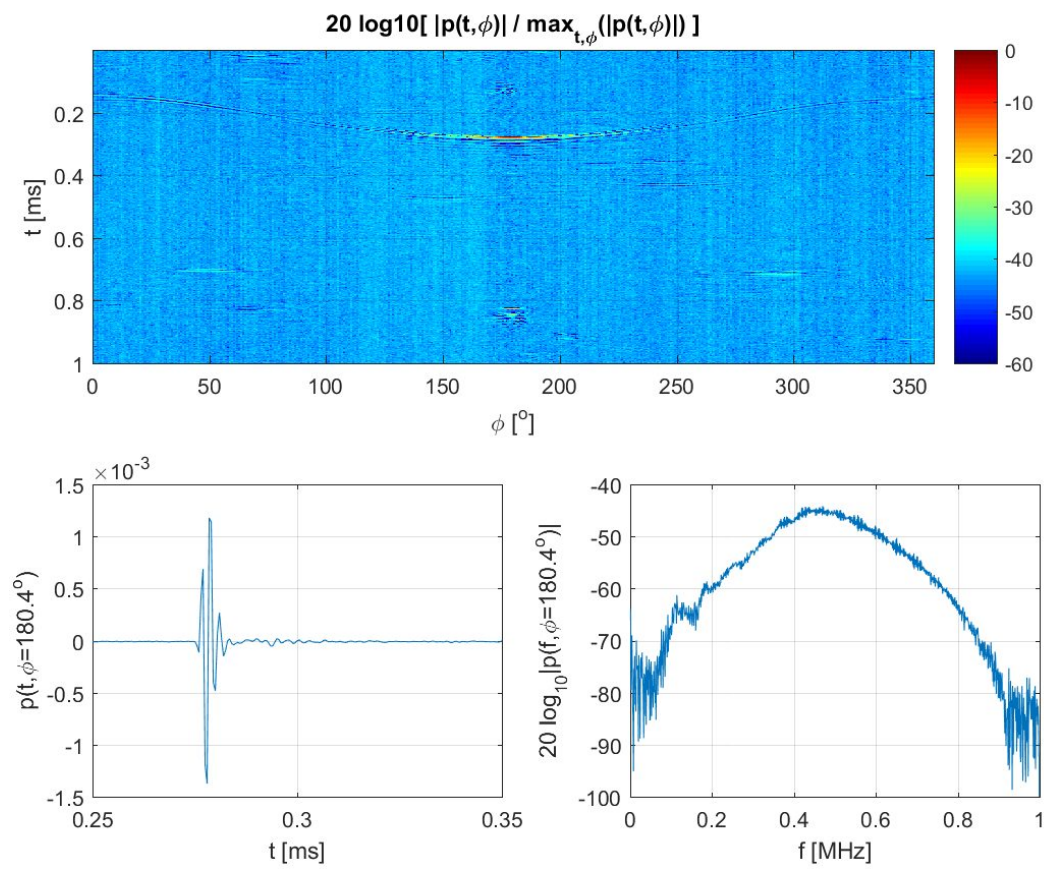

Figure 2: The RF data measured with the DBUS system in absence of an object; (top) B-scan showing the field measured as a function of time and receiver angle for single source position; (bottom-left and -right) single A-scan in time and frequency domain.

with a bandwidth of $1.5 \mathrm{MHz}$ at $-6 \mathrm{~dB}$. The semi-elliptical aperture has a diameter of $26 \mathrm{~cm}$ and a height of $16 \mathrm{~cm}$. Rotational and translational movements, so-called aperture positions, of the complete sensor system create additional virtual positions of the transducers.

The 2041 individual transducers are either operated as emitter (628) or receiver (1413). The transducers have opening angles of $38.2^{\circ}$ (standard deviation $\pm 1.5^{\circ}$ ) at $-6 \mathrm{~dB}$. Four emitters and nine receivers are grouped together including preamplifier and control electronics in so-called Transducer Array Systems (TAS). Each of the 157 TAS contains a temperature sensor for tracking the temperature distribution within the water basin and the shift at each TAS position during measurements. Additionally, two calibrated PT100 temperature sensors are embedded in the TAS holder to enable increased accuracy.

The data acquisition is carried out with an FPGA based system, which can store up to 80 GByte of A-scans [31]. The digitalization is performed by 480 parallel channels (12 Bit at $20 \mathrm{MHz})$, enabling data acquisition at one aperture position in approx. ten seconds. After digitization, the parallel data streams are processed as follows: First, the data streams are bandpass filtered $(1.67$ to $3.33 \mathrm{MHz}$ at $-60 \mathrm{~dB})$. Next, the data rate is reduced by a factor of six by performing bandpass undersampling. Finally, the reduced data is stored in the internal memory buffer. Using this approach up to 47 data sets at different aperture positions can be stored in one data acquisition step. A detailed description of the 3D USCT system can be found in [32].

The emitters are excited with a coded excitation signal, e.g. frequency coded chirps can be applied to increase the signalto-noise ratio of the data. Also the gain of the receiving channels is set individually based on an initial measurement. The applied coded excitation, the individual gain, the temperature data and the spatial positions of the aperture are stored along with the A-scans for each measurement and can be used for signal (pre-) processing and image reconstruction. Empty measurements are also provided and exemplary data is depicted in Figure 3. 

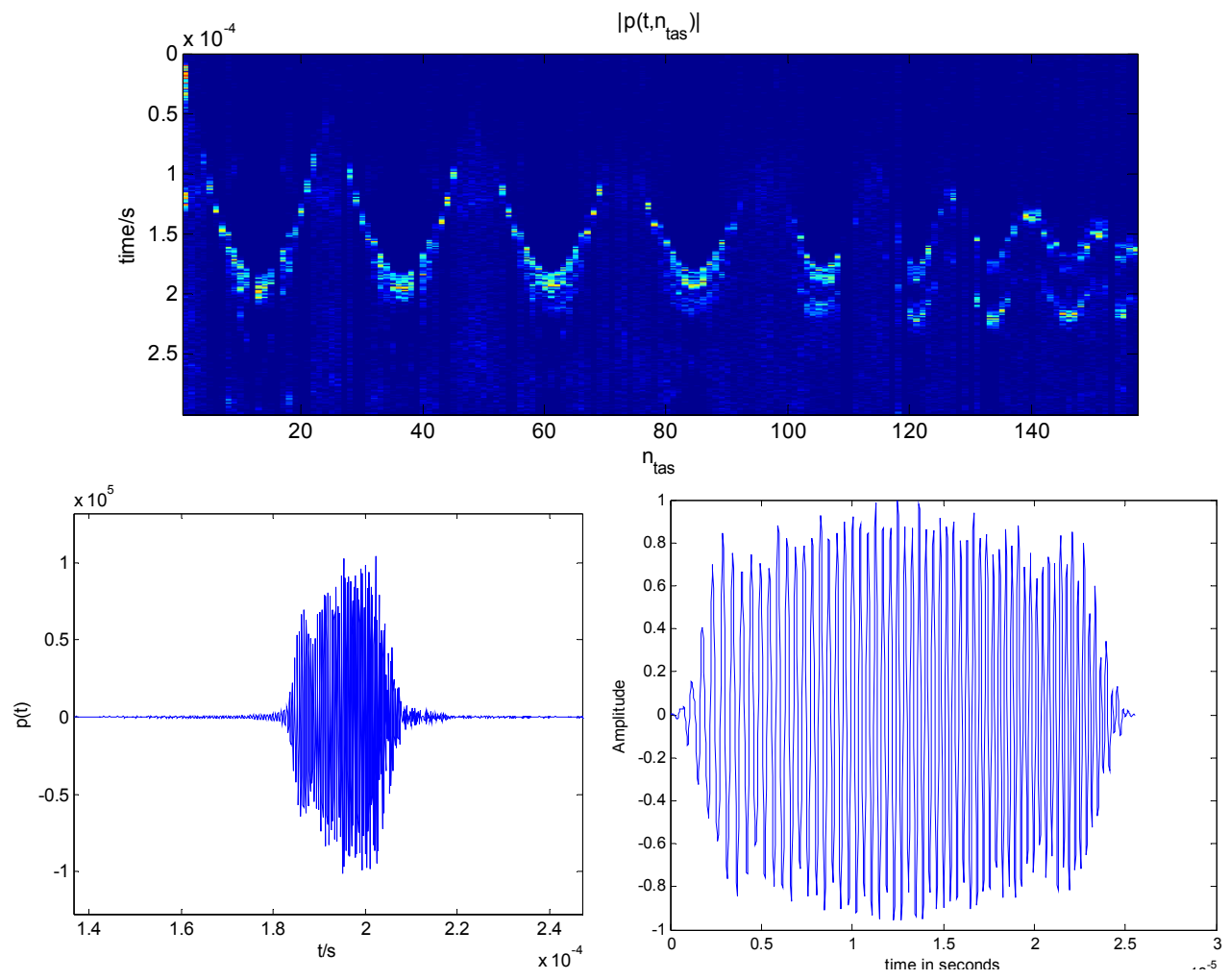

Figure 3: RF data measured with the 3D USCT system in absence of an object; (top) B-scan of the raw data of one emitter in the first TAS of top transducer row of aperture as a function of time and one receiver of each TAS counter-clockwise from top to bottom; bottom-left shows a single A-scan (receiver opposite of emitter element) in time and bottom-right a frequency coded chirp applied for coded excitation.

\section{DATASETS}

In total eight data sets have been made available; two sets are obtained with the system from the TU Delft, and six with the system from KIT. The data is available under "Open Data Commons Attribution License" and the access scripts under the 3-clause BSD license.

A compact overview of the available data sets is presented in Table 1 and Figure 4 shows photos of the phantoms and exemplary reconstructed slices.

\section{TU Delft data sets}

Two data sets are provided by the TU Delft. The first data set is made in absence of an object and can be used as reference measurement. It covers one source and 450 receiver positions equally distributed over $360^{\circ}$. The second data set is an agar based phantom with dimensions of $20 \mathrm{~mm} \times 50 \mathrm{~mm}$, and covers 45 source and 450 receiver positions, all equally distributed over $360^{\circ}$. The tissue mimicking phantom has a volume density of mass of approx. $1004 \mathrm{~kg} / \mathrm{m}^{3}$ and a speed of sound of approx. $1479 \mathrm{~m} / \mathrm{s}$. However, care has to be taken with these values as the conditions under which these values have been obtained may deviate from the actual scanning conditions. The three inclusions were generated by embedding drinking straws in the agar based phantom during curing. Prior to scanning the object, the straws were removed and the inclusions were filled with water.

\section{KIT data set}

Three data sets of different phantoms are provided, each with an empty scan acquired at the same day as the phantom and identical settings of the system's parameters. 

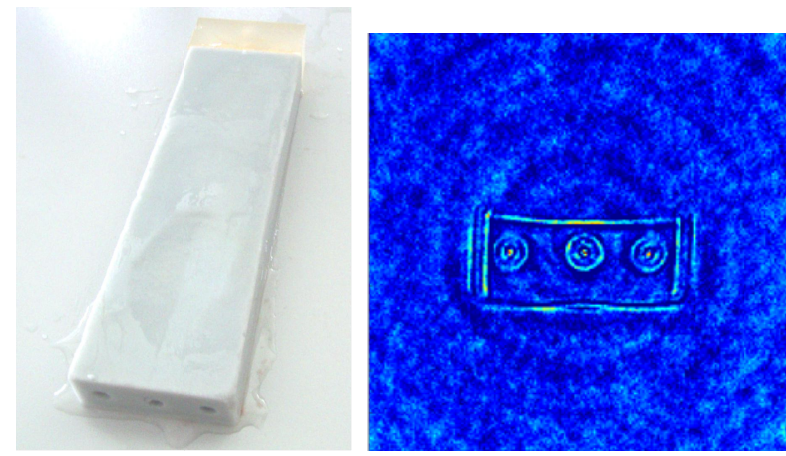

TU Delft agar based phantom
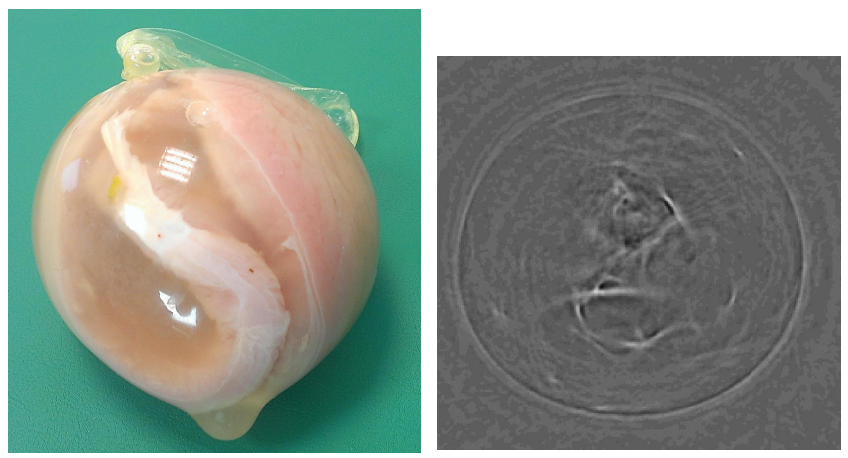

KIT turkey phantom
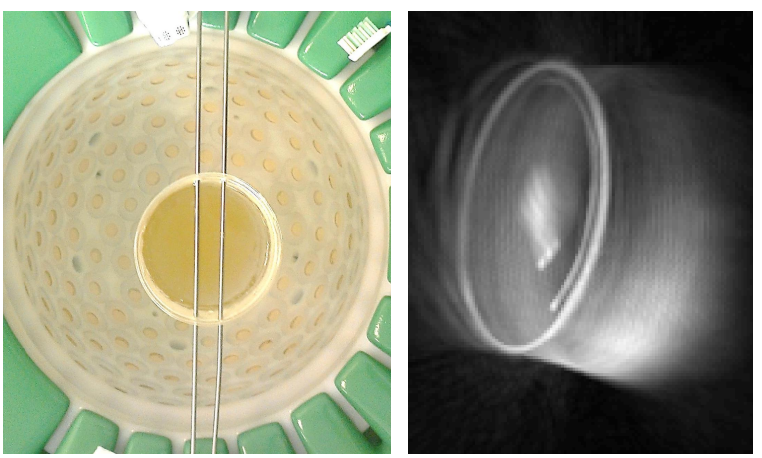

KIT gelatin 3
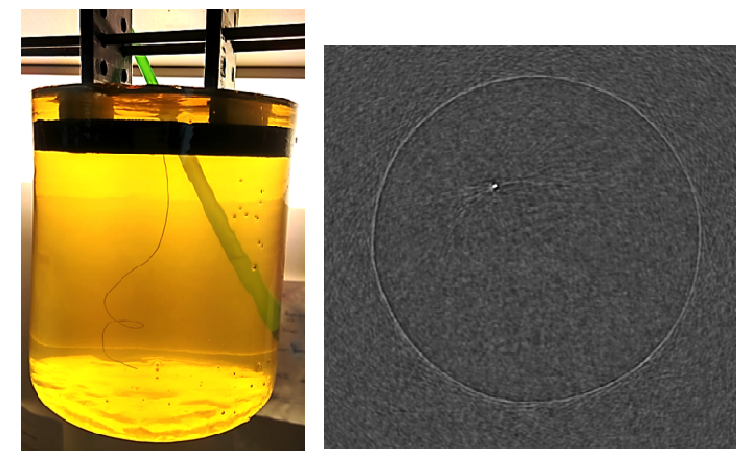

KIT nylon thread phantom

Figure 4: Phantoms scanned; (left) photo, (right) reconstruction using different reconstruction algorithms. From top left to bottom right: TU Delft: agar based phantom reconstructed with Delay and Sum; KIT: gelatin 3 phantom reconstructed with SAFT in low resolution and displayed as maximum intensity projection to enhance the visibility of the bottom of the plastic cup, turkey phantom reconstructed with SAFT, and finally nylon thread phantom with speed of sound corrected SAFT reconstruction.

Gelatin 3: The phantom consists of a gelatin phantom with diameter of approx. $0.07 \mathrm{~m}$ at the bottom and $0.10 \mathrm{~m}$ at the top, a height of approx. $0.13 \mathrm{~m}$ of which approx. $0.10 \mathrm{~m}$ where immersed into the USCT aperture. The speed of sound of the gelatin was approx. $1515 \mathrm{~m} / \mathrm{s}$. The gelatin was embedded in a plastic cup. Two inclusions were generated by embedding drinking straws with diameter $5 \mathrm{~mm}$ in the gelatin during curing. Prior to scanning the object, the straws were removed and the inclusions were filled with water. The phantom was positioned approx. centrally in the 3D USCT aperture.

Turkey phantom: Two olives without stones where embedded into a turkey steak. This steak was then embedded in a condom and filled with gelatin. The resulting phantom has a diameter of approx. $9 \mathrm{~cm}$. The phantom was positioned approx. centrally in the 3D USCT aperture. The turkey steak had an approximate sound speed of $>1550 \mathrm{~m} / \mathrm{s}$ while the olives had a sound speed of approx. $1450 \mathrm{~m} / \mathrm{s}$.

Nylon threads: The phantom consists of a gelatin cylinder with both diameter and height of approx. $10 \mathrm{~cm}$. In this cylinder a nylon thread of diameter $0.2 \mathrm{~mm}$ is embedded. The phantom was centrally positioned in the 3D USCT aperture.

\section{FUTURE PROSPECTIVE FOR USCT REFERENCE DATA BASE}

The aim of this work is to start a freely available USCT data base enabling to test and analyze reconstruction algorithms or to compare the performance of competing algorithms. In a first step initial data base entries are provided and participants of this challenge are asked to report their experiences in using the data. Additionally a panel discussion will 
allow discussing the first steps towards standardized interfaces to access the data of very different USCT systems and brainstorming about specifications for phantoms and real patient data to be included in the data base.

Table 1: Overview of the scans made available.

\begin{tabular}{|c|c|c|}
\hline & TU Delft & KIT \\
\hline Object & None (empty scan) & $\begin{array}{l}\text { None (empty scan) with parameters } \\
\text { fitting gelatin } 3 \text { measurement }\end{array}$ \\
\hline $\begin{array}{l}\text { Center frequency / bandwidth / } \\
\text { chirp length }\end{array}$ & $0.5 \mathrm{MHz} /-$ / - & $2.5 \mathrm{MHz} / 2.67 \mathrm{MHz} / 25.6 \mu \mathrm{s}$ \\
\hline Original sampling rate (stored) & $400 \mathrm{MHz}(400 \mathrm{MHz})$ & $20 \mathrm{MHz}(6.6 \mathrm{MHz})$ \\
\hline $\mathrm{N}_{\text {Aperture pos. }} \times \mathrm{N}_{\text {Source }} \times \mathrm{N}_{\text {Receiver }}$ & $1 \times 1 \times 450$ & $10 \times(157 \times 4) \times(157 \times 9)$ \\
\hline & TU Delft & KIT \\
\hline Object & Agar based tissue mimicking phantom & $\begin{array}{l}\text { Gelatin phantom with two water } \\
\text { inclusions (Gelatin 3) }\end{array}$ \\
\hline $\begin{array}{l}\text { Center frequency / bandwidth / } \\
\text { chirp length }\end{array}$ & $0.5 \mathrm{MHz} /$ - / - & $2.5 \mathrm{MHz} / 2.67 \mathrm{MHz} / 25.6 \mu \mathrm{s}$ \\
\hline Original sampling rate (stored) & $400 \mathrm{MHz}(400 \mathrm{MHz})$ & $20 \mathrm{MHz}(6.6 \mathrm{MHz})$ \\
\hline $\mathrm{N}_{\text {Aperture pos. }} \times \mathrm{N}_{\text {Source }} \times \mathrm{N}_{\text {Receiver }}$ & $1 \times 45 \times 450$ & $10 \times(157 \times 4) \times(157 \times 9)$ \\
\hline & KIT & KIT \\
\hline Object & $\begin{array}{l}\text { None (empty scan) with parameters } \\
\text { fitting Turkey phantom measurement }\end{array}$ & $\begin{array}{l}\text { None (empty scan) with parameters } \\
\text { fitting Nylon threads measurement }\end{array}$ \\
\hline $\begin{array}{l}\text { Center frequency / bandwidth / } \\
\text { chirp length }\end{array}$ & $2.5 \mathrm{MHz} / 2.67 \mathrm{MHz} / 25.6 \mu \mathrm{s}$ & $2.5 \mathrm{MHz} / 2.67 \mathrm{MHz} / 25.6 \mu \mathrm{s}$ \\
\hline Original sampling rate (stored) & $20 \mathrm{MHz}(6.6 \mathrm{MHz})$ & $20 \mathrm{MHz}(6.6 \mathrm{MHz})$ \\
\hline $\mathrm{N}_{\text {Aperture pos. }} \times \mathrm{N}_{\text {Source }} \times \mathrm{N}_{\text {Receiver }}$ & $20 \times(157 \times 4) \times(157 \times 9)$ & $1 \times(157 \times 4) \times(157 \times 9)$ \\
\hline & KIT & KIT \\
\hline Object & $\begin{array}{l}\text { Turkey phantom: Olives embedded in } \\
\text { a turkey steak and gelatin }\end{array}$ & $\begin{array}{l}\text { Nylon thread embedded in gelatin } \\
\text { cylinder }\end{array}$ \\
\hline $\begin{array}{l}\text { Center frequency / bandwidth / } \\
\text { chirp length }\end{array}$ & $2.5 \mathrm{MHz} / 2.67 \mathrm{MHz} / 25.6 \mu \mathrm{s}$ & $2.5 \mathrm{MHz} / 2.67 \mathrm{MHz} / 25.6 \mu \mathrm{s}$ \\
\hline Original sampling rate (stored) & $20 \mathrm{MHz}(6.6 \mathrm{MHz})$ & $20 \mathrm{MHz}(6.6 \mathrm{MHz})$ \\
\hline $\mathrm{N}_{\text {Aperture pos. }} \times \mathrm{N}_{\text {Source }} \mathrm{X} \mathrm{N}_{\text {Receiver }}$ & $20 \times(157 \times 4) \times(157 \times 9)$ & $23 \times(157 \times 4) \times(157 \times 9)$ \\
\hline
\end{tabular}

After the kick off of opening the data base for the public and establishing easy to use data access further challenges are planned, e.g. challenging the different algorithms by comparison of obtained image quality or computational performance. The data sets are freely available and open licensed, so they can also be used outside of challenges for evaluation of advanced reconstruction techniques on real data, further development of algorithms for image reconstruction and signal processing.

The feedback about data and USCT systems resulting from the use with different algorithms can also lead to drive further development of the system architecture, e.g. to research the optimization of transducer positioning or limits on signal-to-noise levels.

We hope also that these challenges will lead to a growth of the data base by increasing the number of imaged objects and/or data acquired with different USCT architectures.

Finally, the overall motivation for all of our work is to support early breast cancer diagnosis. Thus, we also need to learn more about the properties of breast cancer tissues and how to image them to make the most distinguishing properties available for diagnosis. This upcoming knowledge needs to be included into the data base by imaging and if possible quantify the properties of appropriate phantoms and tissue structures. 


\section{ACKNOWLEDGEMENT}

The authors would like to acknowledge the following people: Bastiaan Dekker and Elmer Jansen for building the 2D setup and performing the measurements; Clemens Kaiser, Wei Yap Tan and Carina Wittenbeck for building and measuring phantoms for the 3D USCT and the many colleagues in the institutes who made the built up of USCT systems possible.

\section{REFERENCES}

[1] Ozmen, N.; Dapp, R.; Zapf, M. et al.: "Comparing different ultrasound imaging methods for breast cancer detection," IEEE Transactions on Ultrasonics, Ferroelectrics, and Frequency Control, 62(4), pp. 637-46 (2015).

[2] Natterer, F.: "Acoustic mammography in the time domain," Technical report, University Muenster, (2008).

[3] Zhang, X.; Broschat, S.L.; and Flynn, P.J.: "A numerical study of conjugate gradient directions for an ultrasound inverse problem," Journal of Computational Acoustics, 12(4), pp. 587-604 (2004).

[4] Goncharsky, A.V.; and Romanov, S.Y.: "Supercomputer technologies in inverse problems of ultrasound tomography," Inverse Problems, 29:075004, (2013).

[5] Sandhu, G.Y.; Li; Roy, O.; Schmidt, S.; and Duric, N.: "Frequency domain ultrasound waveform tomography: Breast imaging using a ring transducer," Physics in Medicine and Biology, 60(14), pp. 5381-98 (2015).

[6] Wiskin, J.; Borup, D.; Johnson, S.; et al.: "Three dimensional nonlinear inverse scattering: Quantitative transmission algorithms, refraction corrected reflection, scanner design and clinical results," Proc. Meetings on Acoustics, 19, (2013).

[7] Chenevert, T.L.; Bylski, D.I.; Carson, P.L.; et al.: "Ultrasonic computed tomography of the breast. Improvement of image quality by use of cross-correlation time-of-flight and phase-insensitive attenuation measurements," Radiology, 152 (1), pp. 155-9 (1984).

[8] Kaveh, M.; Mueller, R.K.; Rylander, R.; et al.: "Experimental results in ultrasonic diffraction tomography," Acoustical Imaging (K.Y. Wang, Ed.), 9, pp. 433-50 (1980).

[9] Simonetti, F.; Huang, L.; and Duric, N.: "A multiscale approach to diffraction tomography of complex threedimensional objects," Applied Physics Letters, 95, (2009).

[10] Sponheim, N.; Gelius, L.-J.; Johansen, I.: and Stamnes, J.J.: "Quantitative results in ultrasonic tomography of large objects using line sources and curved detector arrays," IEEE Transactions on Ultrasonics, Ferroelectrics, and Frequency Control, 38(4), pp. 370-9 (1991).

[11] Greenleaf, J.F.; Ylitalo, J.; and Gisvold, J.J.: "Ultrasonic computed tomography for breast examination," IEEE Engineering in Medicine and Biology Magazine: The Quarterly Magazine of the Engineering in Medicine \& Biology Society, 6(4), pp. 27-32 (1987).

[12] Andre, M.P.; Janee, H.S.; Martin, P.J.; et al.: "High speed data acquisition in a diffraction tomography system employing large-scale toroidal arrays," International Journal of Imaging Systems and Technology, 8(1), pp. 137-47 (1997).

[13] Lavarello, R.J.; and Oelze, M.L.: "Tomographic reconstruction of three-dimensional volumes using the distorted Born iterative method," IEEE Transactions on Medical Imaging, 28(10), pp. 1643-53 (2009).

[14] Andersen, A.H.: "A ray tracing approach to restoration and resolution enhancement in experimental ultrasound tomography," Ultrasonic Imaging, 12(4), pp. 268-91 (1990).

[15] Hormati, A.; Jovanovic, I.; Roy, O.; and Vetterli, M.: "Robust ultrasound travel-time tomography using the bent ray model" Proc. SPIE 7629, Medical Imaging 2010: Ultrasonic Imaging, Tomography, and Therapy, 7629, (2010).

[16]Li, C.; Duric, N.; and Huang, L.: "Breast imaging using transmission ultrasound: Reconstructing tissue parameters of sound speed and attenuation," BioMedical Engineering and Informatics: New Development and the Future Proceedings of the 1st International Conference on BioMedical Engineering and Informatics, 2, pp. 708-12 (2008).

[17]Dapp; R.: "Abbildungsmethoden für die Brust mit einem 3D-Ultraschall-Computertomographen," PhD thesis, Karlsruhe Institute of Technology, (2013).

[18] Kretzek, E.; and Ruiter, N.V.: "GPU based 3D SAFT reconstruction including phase aberration," Proc. SPIE 9040, Medical Imaging 2014: Ultrasonic Imaging and Tomography, p. 90400W (2014).

[19] Kretzek, E.; Hopp, T.; and Ruiter, N.V.: "GPU-based 3D SAFT reconstruction including attenuation correction," Proc. SPIE 9419, Medical Imaging 2015: Ultrasonic Imaging and Tomography, p. 94190E, (2015). 
[20] Kretzek, E.; Hucker, P.; Zapf, M.; and Ruiter, N.V.: "Evaluation of directional reflectivity characteristics as new modality for 3D Ultrasound Computer Tomography," 2015 IEEE International Ultrasonics Symposium (IUS), (2015).

[21] Ramirez, A.B., and van Dongen K.W.A.: "Sparsity Constrained Contrast Source Inversion," Journal of Acoustical Society of America, 140(3), pp. 1749-57 (2016).

[22] van Dongen, K.W.A.; and Wright, W.M.D.: "A full vectorial Contrast Source inversion scheme for 3D acoustic imaging of both compressibility and density profiles," Journal of the Acoustical Society of America, 121(3), pp. 1538-49 (2007).

[23] van Dongen, K.W.A.; and Wright, W.M.D.: "A forward model and conjugate gradient inversion technique for lowfrequency ultrasonic imaging," Journal of the Acoustical Society of America 120(4), pp. 2086-95 (2006).

[24] Rouyer, J., Mensah, S., Franceschini, E., et al.: "Conformal ultrasound imaging system for anatomical breast inspection," IEEE Trans UFFC, 59(7), pp. 1457-69 (2012).

[25] Wiskin, J., Borup, D., Callahan, K., et al.: "Inverse scattering theory,” In Acoustical Imaging. (M. P. André, J. P. Jones, \& H. Lee, Eds.), 30, (2011).

[26] Marmarelis, V.Z.; Jeong, J.; Shin, D.C. and Do, S.: "High-resolution 3-D imaging and tissue differentiation with transmission tomography," Acoustical Imaging (Vol. 28), pp. 195-206 (2007).

[27] Duric, N., Littrup, P., Schmidt, S.; et al.: "Breast imaging with the SoftVue imaging system: first results," In Proc. of SPIE, 8675, p. 86750K-86750K-8 (2013).

[28] Ruiter, N. V, Göbel, G., Berger, L., et al.: "Realization of an optimized 3D USCT," In J. D’hooge \& M. M. Doyley (Eds.), Proc. of SPIE, pp. 796805-796805-8 (2011).

[29] http://ipeusctdb1.ipe.kit.edu/ usct/challenge/

[30] https://opensource.org/licenses/BSD-3-Clause

[31] Kopmann, A.; Bergmann, T.; Gemmeke, H.; et al.: "FPGA-based DAQ system for multi-channel detectors," Proc. IEEE NSS MIC, (2008).

[32] Ruiter, N.V., Zapf, M., et al.: "First Results of a Clinical Study with 3D Ultrasound Computer Tomography," Proc. IEEE Internat. Ultrasonics Symp., (2013).

[33] Max Planck Society: "Berlin Declaration on Open Access to Knowledge in the Sciences and Humanities," (2003). https://openaccess.mpg.de/Berlin-Declaration

[34] G7 Science and Technology Ministers' Meeting: "Tsukuba Communiqué”, (2016). http://www8.cao.go.jp/cstp/english/others/20160517communique.pdf

[35] Helmholtz Gemeinschaft: „Positionspapier zum Umgang mit Forschungsdaten in der Helmholtz-Gemeinschaft,“ (2016). https://www.helmholtz.de/fileadmin/user_upload/01_forschung/Open_Access/DE_AKOS_TG-

Forschungsdatenleitlinie_Positionspapier.pdf 